\title{
Ionic liquid synthesis in a microstructured reactor for process intensification
}

\author{
A. Renken ${ }^{b, *}$, V. Hessel ${ }^{\mathrm{a}, \mathrm{c}}$, P. Löb ${ }^{\mathrm{a}}$, R. Miszczuk ${ }^{\mathrm{b}}$, \\ M. Uerdingen ${ }^{d}$, L. Kiwi-Minsker ${ }^{b}$ \\ ${ }^{a}$ Institut für Mikrotechnik Mainz, GmbH, Carl-Zeiss-Str. 18-20, D-55129 Mainz, Germany \\ ${ }^{\mathrm{b}}$ Ecole Polytechnique Fédérale de Lausanne, SB-ISIC-GGRC-Station 6, CH-1015 Lausanne, Switzerland \\ ${ }^{\mathrm{c}}$ Eindhoven University of Technology, P.O. Box 513, $5600 \mathrm{MB}$ Eindhoven, The Netherlands \\ d Solvent Innovation GmbH, Nattermannallee 1, D-50829 Köln, Germany \\ Received 24 May 2007; accepted 28 May 2007 \\ Available online 15 June 2007
}

\begin{abstract}
Ionic liquids (IL) are the focus of growing interest over the last few years due to their low vapour pressure being beneficial for replacing common organic solvents with high vapour pressure. IL synthesised via alkylation are produced in batch or semi-batch stirred tank reactors. The reaction is highly exothermic and the kinetics was shown to be fast. The heat management during the reactor operation is a crucial point leading to high quality IL product and avoiding thermal runaway. This study reports the use of a microstructured reactor (MSR) system for the production of ethylmethylimidazole ethylsulfate by a solvent-free alkylation reaction. A combination of MSR and two tubular capillary reactors operating at two different cooling temperatures has been proposed. The save and stable operation of this reactor system is proven experimentally rendering the IL of high quality. The specific reactor performance was about $4 \mathrm{~kg} \mathrm{~m}^{-3} \mathrm{~s}^{-1}$ being ca. 3 orders of magnitude higher as compared to more traditional reactors.
\end{abstract}

(C) 2007 Elsevier B.V. All rights reserved.

Keywords: Microreactor; Process intensification; Novel chemistry; Ionic liquids

\section{Introduction}

Ionic liquids (IL) have gained an enormous interest over the past years due to their unique low vapour pressure being beneficial for the replacement of traditional organic solvents in organic synthesis and extraction [1,2]. The potential applications of IL may lead to an increasing demand. Therefore, appropriate methods allowing their production on industrial scale are warranted. Traditionally, IL synthesised via alkylation are produced in batch or semi-batch stirred tank reactors. The reaction is highly exothermic and the kinetics was shown to be fast. The heat management is of major concern to attain high quality product and to avoid thermal runaway. Therefore, it is difficult to increase the specific reactor performances by using traditional reactors. In the present study, we report the use of microstructured reactors (MSR) for the production of

\footnotetext{
* Corresponding author.

E-mail address: albert.renken@epfl.ch (A. Renken).
}

an ethylmethylimidazole ethylsulfate ([EMIM] $\left[\mathrm{EtSO}_{4}\right]$ under solvent-free conditions via alkylation of methylimidazole with diethylsulfate. Organic synthesis under solvent-free conditions leads to more benign processes and is an important factor for process intensification.

The scheme of the chosen reaction is presented in Fig. 1.

Chemical microstructured reactors (MSR) are devices containing open paths for fluids with dimensions in the submillimeter range. Mostly MSR have multiple parallel channels with diameters between 10 and several $100 \mu \mathrm{m}$ where the chemical transformations occur. This gives a high specific surface area in the range of $10,000-50,000 \mathrm{~m}^{2} \mathrm{~m}^{-3}$ and allows an effective mass and heat transfer compared to traditional chemical reactors. Another important feature of MSR is that the heat exchange and the reaction are often performed in the same gadget. MSR are operated under laminar flow with the heat transfer coefficient for liquids about $10 \mathrm{~kW} \mathrm{~m}^{-2} \mathrm{~K}^{-1}$. This is one order of magnitude higher than in the traditional heat exchangers and allows: to avoid hot-spot formation, to attain higher reaction temperatures and to reduce reaction volumes. This in 


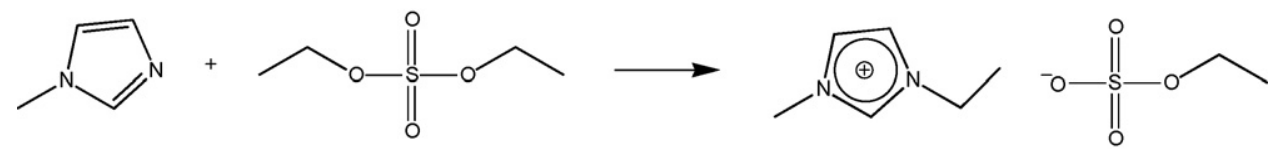

Fig. 1. Synthesis of the ionic liquid 1-ethyl-3-methylimidazolium ethylsulfate [EMIM][EtSO 4 .

turn improves the energy efficiency and reduces the operational cost.

The chosen alkylation reaction is highly exothermic. Precise temperature control is required to achieve high quality products and to prevent temperature runaway of the reactor. Thus, heat removal is an important concern for the reactor design and operation. The common reactor type is the semi-batch stirred tank reactor where the dosage of one reactant is adapted to the heat transfer in the reactor. This leads to large reactor volumes and poor specific reactor performances.

The use of a continuously operated tubular reactor was recently proposed for this reaction $[3,4]$. To increase the specific heat exchange area, tubes with diameters in the millimetre range were used. But even in reactors with $6 \mathrm{~mm}$ inner diameter, pronounced axial temperature profiles were observed and cooling temperatures of $40^{\circ} \mathrm{C}$ or higher lead to thermal runaway [3]. When working at low temperatures, long residence times of several hours or even days are necessary to achieve high conversions [5].

Due to the high reactant concentration, the highest reaction rate and heat production is near the reactor entrance and the temperature is difficult to control. Moreover, fast and efficient mixing of the reactants is also very important. We, therefore, propose to use microstructured devices for an efficient mixing and heat removal at the entrance of the reactor system. The following sections of the reactor are less critical. This allows switching to a millimetre, finally to a centimetre scale. In addition, temperatures can be adapted to the reaction kinetics to get high overall transformation rates. This multiscale reactor design is a powerful tool for the optimisation of the reactor performance [6].

The maximum acceptable reaction temperature to ensure the high quality of the product has been determined in separate experiments. In addition, calorimetric measurements were performed to get kinetic and thermodynamic data required for the design of the reactor system [7].

\section{Experimental}

Both reactants are supplied by Sigma-Aldrich: methyl imidazole puriss. GC (>99\%) and diethyl sulfate purum GC (>99\%). Initial concentrations for both reactants are kept identical at $4.8 \mathrm{kmol} \mathrm{m}^{-3}$, if not specified otherwise. The ionic liquid 1-ethyl-3-methylimidazolium ethylsulfate was obtained from Solvent Innovation.

\subsection{Decomposition studies}

Decomposition studies were performed with both reactants as well as the final product to check their thermal stabilities.
The aim was to determine the maximum reaction temperature at which a colorless product can be obtained.

The experiments were performed by heating up the reaction partners in a temperature range from $20^{\circ} \mathrm{C}$ up to $170^{\circ} \mathrm{C}$. Each component was transferred into a double-necked, roundbottomed flask equipped with reflux condenser and stirrer and then heated up in an oil bath with a temperature ramp of about $4 \mathrm{~K}_{\mathrm{min}^{-1}}$.

\subsection{Reaction kinetics and reaction enthalpy}

The reaction kinetics was studied by differential scanning calorimetry (DSC; Mettler) at temperatures well below the maximum value. Therefore, decomposition reactions can be neglected and the heat signal corresponds to the rate of the IL formation. Experiments were carried out under isothermal as well as non-isothermal conditions by imposing a well-defined temperature ramp to the samples [8]. The reaction starts already at room temperature. Therefore, the reactants were diluted with the final product to slow down the rate, thus reducing the error due to uncontrolled transformation within the stabilization period of the DSC.

The reaction enthalpy $\left(\Delta H_{\mathrm{r}}\right)$ can be determined by integration of the experimentally obtained heat flux $(\dot{Q})$ signal assuming heat effects due to mixing and/or phase change negligible.

$-\Delta H_{\mathrm{r}}=\frac{Q_{\mathrm{tot}}}{n} \quad$ with $\quad Q_{\mathrm{tot}}=\int_{t=0}^{t=\infty} \dot{Q} \mathrm{~d} t$

where $\Delta H_{\mathrm{r}}$ is the total enthalpy change $\left(\mathrm{kJ} \mathrm{kmol}^{-1}\right)$ and $n$ the total number of moles in the crucible reacted.

\subsection{Analysis}

The reactants were analyzed by liquid chromatography on a column with a non-polar stationary phase and an UV detector. Samples at the reactor outlet were collected into a flask containing an aqueous buffer solution. As ethylsulfate reacts immediately with water the reaction is quenched and the mixture can be analyzed off-line.

\subsection{Microreactor setup}

The setup is shown in Fig. 2. The reactants were dosed by syringe pumps and mixed in a small Caterpillar micromixer with channel dimensions of $600 \mu \mathrm{m} \times 600 \mu \mathrm{m}$ (Institut für Mikrotechnik Mainz, Fig. 3). The mixer was kept at room temperature. As first reactor a microstructured multichannel reactor (R1) was used. This microstructured reactor (MSR) is composed of a stack of plates with parallel micro-channels. The chan- 


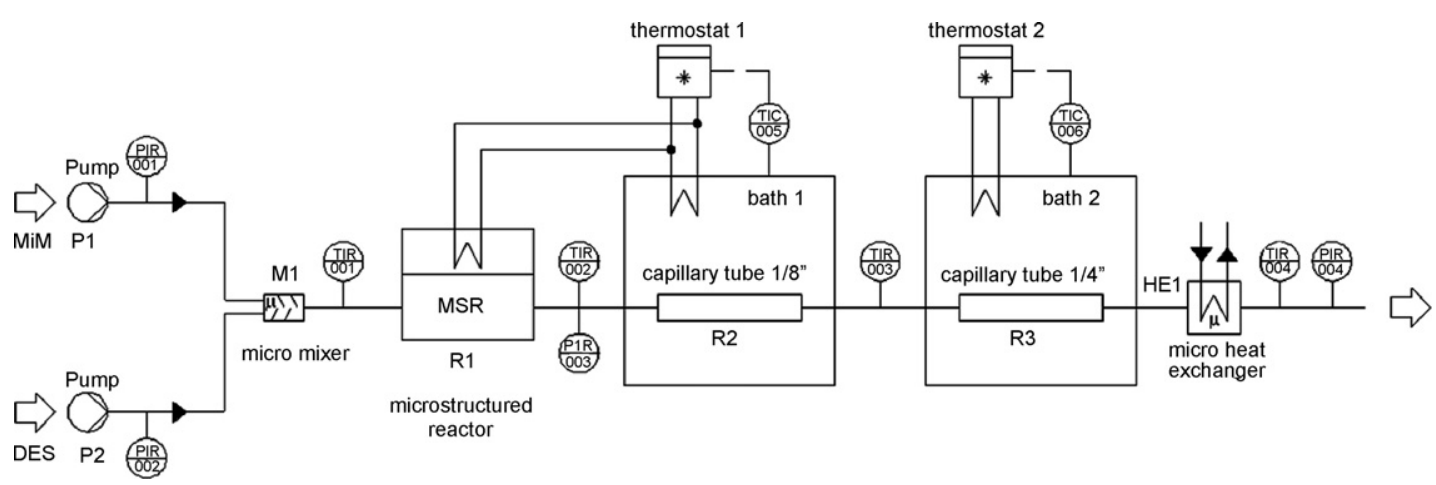

Fig. 2. Scheme of the experimental setup.

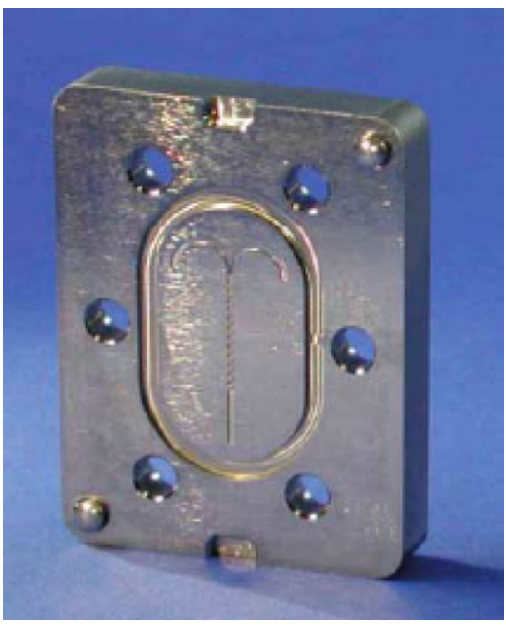

Fig. 3. Photo of the Caterpillar micromixer, $600 \mu \mathrm{m} \times 600 \mu \mathrm{m}$ (IMM, Mainz).

nels are rectangular: $1 \mathrm{~mm}$ wide and $0.65 \mathrm{~mm}$ high. The channel length is $125 \mathrm{~mm}$. The reactant are distributed over the channels in an entrance chamber and collected at the end in a chamber of similar design (Fig. 4). All plates are welded together (laser welding) on the external sides, and are kept together by adding an external cover (plain PTFE) on each side to apply constant pressure in the middle of the plates.

In total four plates are assembled in the sequence cooling-reaction-reaction-cooling. The total inner volume for the reaction plates (including entrance and outlet chambers) is $10.8 \mathrm{~cm}^{3}$

The MSR is connected to a tubular $1 / 8$ in. reactor (R2) with an inner diameter of $d_{\mathrm{i}}=1.75 \mathrm{~mm}$ and a volume of $6.70 \mathrm{~cm}^{3}$.
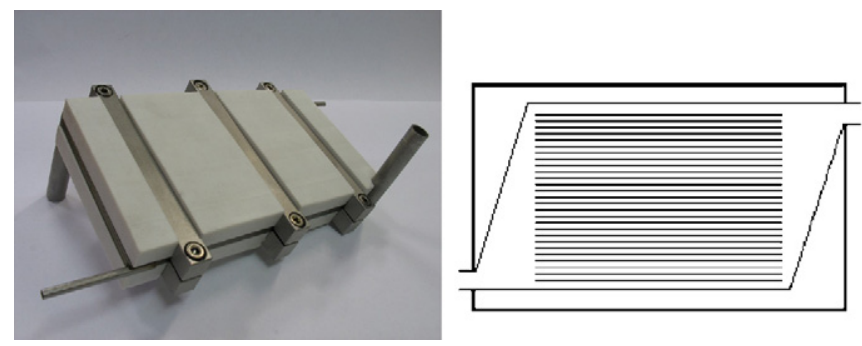

Fig. 4. Photo of the microstructured reactor and scheme of a single plate (IMM, Mainz).
MSR and R2 are connected to the same thermostat and thus are operated at the same cooling temperature. The 3rd reactor (R3) consists of a $1 / 4 \mathrm{in}$. tube with an inner diameter of $d_{\mathrm{i}}=4.37 \mathrm{~mm}$ and a reactor volume of $13.8 \mathrm{~cm}^{3}$ embedded in a second bath with higher temperature of 343 or $368 \mathrm{~K}$. The reaction mixture at the reactor outlet is cooled down to room temperature in a heat exchanger.

\section{Results and discussion}

\subsection{Decomposition studies}

From the three substances tested, only methyl-imidazole exhibits a color change when heated. It was shown that the coloration starts at temperatures above $100{ }^{\circ} \mathrm{C}$. The color gets more yellowish while heating and even turns to orange at about $170^{\circ} \mathrm{C}$. Therefore, a special care has to be taken to limit the reaction temperature at $100^{\circ} \mathrm{C}$ to avoid coloration.

\subsection{Reaction enthalpy}

The reaction enthalpy has been determined from calorimetric experiments with reactants diluted with the final product. A dilution of a factor 10 was found to be necessary to get reproducible results of $\Delta H_{\mathrm{r}}=-100 \pm 2\left[\mathrm{~kJ} \mathrm{~mol}^{-1}\right]$. This result is in line with published values [3].

\subsection{Reaction kinetics}

Kinetic parameters were estimated by fitting the measured calorimetric data to a model for an irreversible $n$th order kinetics (Eq. (2)).

$r=k C^{n} ; \quad k=k_{0} \exp \left(-\frac{E_{\mathrm{a}}}{R T}\right)$

In total twelve experimental measurements under isothermal and non-isothermal conditions were carried out. The temperature ramp was varied between 3 and $8 \mathrm{~K} \mathrm{~min}^{-1}$. An example is shown on Fig. 5 for a temperature ramp of $5 \mathrm{~K} \mathrm{~min}^{-1}$. Fitting parameters are:

reaction order: $n=2$

activation energy: $E_{\mathrm{a}}=86 \mathrm{~kJ} \mathrm{~mol}^{-1}$

rate constant at $T=303 \mathrm{~K}: k_{303}=1.9 \times 10^{12} \mathrm{~m}^{3} \mathrm{kmol}^{-1} \mathrm{~s}^{-1}$ 


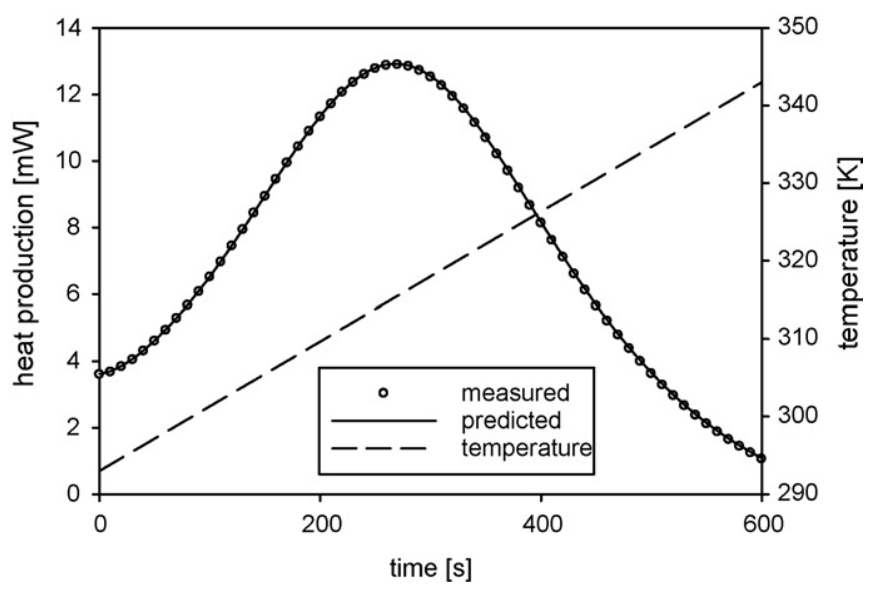

Fig. 5. Measured and predicted heat production. DSC measurement, temperature ramp: $5 \mathrm{~K} \mathrm{~min}^{-1}$, start temperature: $293 \mathrm{~K}$.

\subsection{Heat exchange performance of the MSR}

As the heat transfer coefficient will be different in the channel and the in- and outlet region, the heat exchange performance of the MSR in counter- and co-current mode was determined in separate experiments. The product of the overall heat transfer coefficient $(U)$ and the effective heat exchange area follows from the energy balance.

$U A=\frac{\dot{Q}_{\mathrm{ex}}}{\Delta T_{\mathrm{m}}} ; \quad$ with $\quad \Delta T_{\mathrm{m}}=\frac{\Delta T_{\mathrm{in}}-\Delta T_{\text {out }}}{\ln \left(\Delta T_{\mathrm{in}} / \Delta T_{\text {out }}\right)}$

From different experiments at varied flow rates and temperatures the following result was obtained: $U A=0.65 \mathrm{~kW} \mathrm{~K}^{-1}$.

\subsection{Maximum cooling temperature for stable reactor operation}

The risk of temperature runaway with hot spot formation corresponding to the adiabatic temperature raise is particular high at the entrance of the first reactor, due to the high reactant concentrations. In addition, the reactor may become very sensitive to small parameter variations as cooling temperature, flow rate or cooling performance. This domain of high "parametric sensitivity" [9] must be avoided for stable reactor operation.

For given reaction kinetics the thermal behaviour of a tubular reactor depends on three different parameters [10]:

The Arrhenius number:

$\gamma=\frac{E_{\mathrm{a}}}{R T_{\mathrm{c}}}$

The heat production potential:

$S^{\prime}=\frac{\Delta T_{\mathrm{ad}}}{T_{\mathrm{c}}} \cdot \frac{E_{\mathrm{a}}}{R T_{\mathrm{c}}}$

The ratio of the characteristic reaction time to the cooling time:

$N=\frac{t_{\mathrm{r}}}{t_{\mathrm{c}}}=\frac{1}{k\left(T_{\mathrm{c}}\right) c_{10}^{n-1}} \frac{U A}{\rho c_{\mathrm{p}} V_{\mathrm{r}}}$
For a given heat production potential, $S^{\prime}$, the axial temperature profile is strongly influenced by the ratio between the characteristic reaction and cooling time. The minimum ratio $t_{\mathrm{r}} / t_{\mathrm{c}}=N_{\min }$ for a stable reactor operation as function of the heat production potential can be estimated with the following empirical relation $[11,12]$.

$N_{\min }=\left(\frac{t_{\mathrm{r}}}{t_{\mathrm{c}}}\right)_{\min }=2.72 S^{\prime}-B \sqrt{S^{\prime}}$

The parameter $B$ is dependent on the reaction order, $n$, and is found to be $B=0(n=0), B=3.37(n=1), B=4.57(n=2)$.

Respecting Eq. (7) allows keeping the maximum temperature (hot spot) independent of the adiabatic temperature raise for constant Arrhenius number.

The conversion in the micromixer was found to be about $1 \%$, which reduces the inlet concentration for the MSR to $4.75 \mathrm{kmol} \mathrm{m}^{-3}$. Supposing a specific heat of $c_{\mathrm{p}}=2.54 \mathrm{~kJ} \mathrm{kmol}^{-1} \mathrm{~K}^{-1}$ and a density of $\rho=1228 \mathrm{~kg} \mathrm{~m}^{-3}$ (Solvent Innovation), the parameters $N$ and $S^{\prime}$ can be estimated with the measured kinetics and the heat transfer performance of the MSR as a function of the cooling temperature (Fig. 6).

As seen from Fig. 6, stable operation of the MSR can be expected for cooling temperatures of $T_{\mathrm{c}} \leq 323 \mathrm{~K}$. For $T_{\mathrm{c}}=323 \mathrm{~K}$ a hot spot temperature of about $335 \mathrm{~K}$ is estimated [11], which is far below the maximum acceptable value of $373 \mathrm{~K}$.

\subsection{Reactor performance}

The hydrodynamic characteristics of the MSR is not correctly known, therefore we renounced to simulate its behaviour. For the following capillary reactors, temperature and concentration profiles were calculated, assuming plug flow behaviour. The resulting final conversions are compared to measured values.

Laminar flow and a fully developed temperature profile is assumed for the capillary reactor. Under these conditions, the

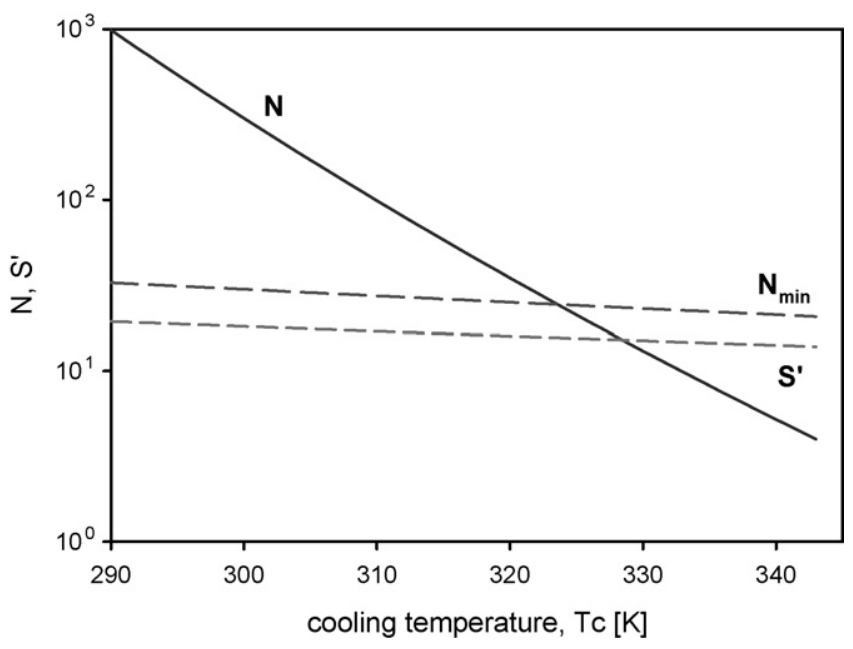

Fig. 6. Stability parameter for the MSR as function of cooling temperature. 


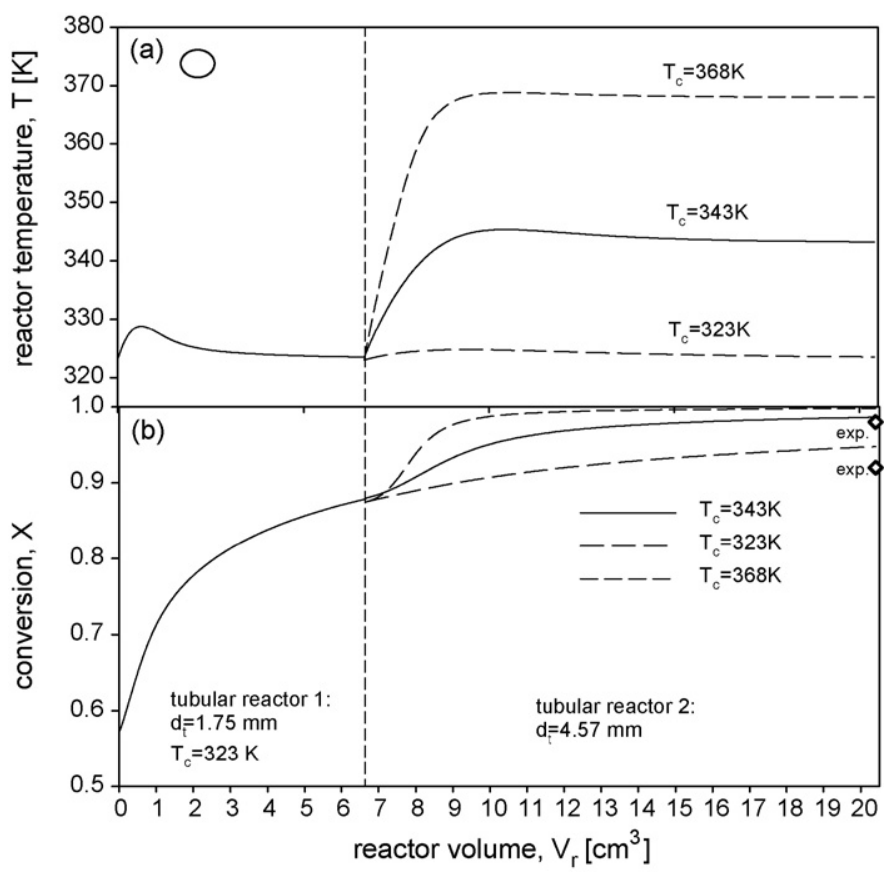

Fig. 7. (a) Temperature and (b) conversion profile in the capillary reactors.

Nusselt number, $N u$, reaches an asymptotic value, $N u_{\infty}$ (see Eq. (8)

$N u=\frac{h d_{\mathrm{t}}}{\lambda} ; \quad N u_{\infty}=3.66($ circular tube $)$

where $\lambda$ is the heat conductivity of the reaction mixture and $h$ is the heat transfer coefficient.

To simplify the analysis, we will neglect the heat transfer resistance in the reactor wall. As only a single reaction takes place in the reactor, the steady-state temperature profile of the fluid can be calculated by solving the following mass and heat balances by numerical integration [13].

$\frac{\mathrm{d} T}{\mathrm{~d} V}=\frac{\left(-R_{1}\right)\left(-\Delta H_{\mathrm{r}}\right)}{\dot{Q} \rho \bar{c}_{\mathrm{p}}}-\frac{h 4\left(T-T_{\mathrm{c}}\right)}{d_{\mathrm{t}} \dot{Q} \rho \bar{c}_{\mathrm{p}}}$

$\frac{\mathrm{d} X}{\mathrm{~d} V}=\frac{-R_{1}}{\dot{Q} C_{1,0}}$

Experiments were carried out at constant flow rate of $7 \mathrm{~cm}^{3} \mathrm{~min}^{-1}$ and a cooling temperature of $T_{1}=323 \mathrm{~K}$. Under these condition a conversion of $X_{\mathrm{MSR}}=0.57$ in the MSR is obtained. The conversion in the first capillary reactor increases to about $X_{\mathrm{R} 1}=0.87$. Only a moderate maximum temperature peak of about $6 \mathrm{~K}$ is predicted (Fig. 7a). The second capillary reactor with an inner diameter of $4.57 \mathrm{~mm}$ is used to complete the conversion. Experiments were performed at temperatures $T_{\mathrm{c}}=323$ and $343 \mathrm{~K}$. The predicted conversions are close to the measured one (Fig. 7b):

$X_{\mathrm{R} 2,323}=0.946$ (measured $: X_{\mathrm{ex}}=0.92$ )

$X_{\mathrm{R} 2,343}=0.986$ (measured : $X_{\mathrm{ex}}=0.98$ ).
After heating up of the reaction mass, the reactor has a quasi isothermal behaviour. In consequence, the temperature can be kept close to the maximal allowable. This is shown in Fig. 7 for $T_{\mathrm{c}}=368 \mathrm{~K}$. A final conversion of $X_{\mathrm{R} 2,368}=0.998$ is predicted.

With the chosen volumetric flow rate of $Q=7 \mathrm{~cm}^{3} \mathrm{~min}^{-1}$ a performance of about $2 \mathrm{molh}^{-1}$ corresponding to about $0.5 \mathrm{~kg} \mathrm{~h}^{-1} \mathrm{IL}$ can be obtained. The space time for the reactors is $4.6 \mathrm{~min}$, this corresponds to a specific performance of $4.4 \mathrm{~kg} \mathrm{~m}^{-3} \mathrm{~s}^{-1}$

\section{Conclusion}

A first prototype of a reactor system composed of a microstructured reactor in combination with two tubular capillary reactors with diameters in the range of several millimetres has been developed and tested for a total flow rate of $0.41 \mathrm{~h}^{-1}$. Compared to simple capillary reactors operating at two different temperature levels, the space time was reduced from ca. $4 \mathrm{~h}$ to $4.6 \mathrm{~min}$, which corresponds to the performance increase of a factor of 50. Compared to isothermal operation an increase of 3 orders of magnitude is achieved. Further improvements can be expected by increasing the diameter of the first capillary reactor.

The feasibility of this novel reactor system is proven and the set-up is now under long-time testing by an industrial partner. The throughput capacity could be adjusted to the demand of the user by numbering-up [14].

\section{References}

[1] M. Wagner, Synthesis and catalysis in ionic liquids, Chim. Oggi 22 (6) (2004) 17-19.

[2] P. Wasserscheid, T. Welton, Ionic Liquids in Synthesis, Wiley-VCH, Weinheim, 2004.

[3] A. Große-Böwing, A. Jess, P. Wasserscheid, Kinetik und Reaktionstechnik der Synthese ionischer Flüssigkeiten, Chem. Ing. Techn. 77 (2005) 1430-1439.

[4] P. Löb, V. Hessel, U. Krtschil, H. Löwe, Continuous micro-reactor rigs with capillary sections in organic synthesis: Generic process flow sheets, practical experience, and "Novel Chemistry", Chim. Oggi 24 (2) (2006) $46-50$.

[5] A. Große-Böwing, A. Jess, Kinetics and reactor design aspects of the synthesis of ionic liquids-experimental and theoretical studies for the ethylmethylimidazole ethylsulfate, Chem. Eng. Sci. 62 (2007) 17601769.

[6] V. Hessel, P. Löb, H. Löwe, Development of microstructured reactors to enable organic synthesis rather than subduing chemistry, Curr. Org. Chem. 9 (8) (2005) 765-787.

[7] R. Miszczuk, Master thesis: Multi-Scaled Reactor Design for the Synthesis of the Ionic Liquid [EMIM][EtSO ${ }_{4}$ ] Using Micro Process Engineering, 2007, Ecole polytechnique fédérale de Lausanne.

[8] F. Stoessel, Applications of reaction calorimetry in chemical engineering, J. Therm. Anal. 49 (3) (1997) 1677-1688.

[9] O. Bilous, N.R. Amundson, Chemical reactor stability and sensitivity.II, AIChE J. 2 (1956) 117-126.

[10] C.H. Barkelew, Stability of chemical reactors, AIChE Symp. Ser. 55 (25) (1959) 38-46.

[11] M. Baerns, A. Renken, Chemische Reaktionstechnik, in WinnackerKüchler: Chemische Technik: Prozesse und Produkte, Wiley-VCH, Weinheim, 2004, 453-643. 
[12] A. Renken, L. Kiwi-Minsker, in: N. Kockmann (Ed.), Chemical Reactions in Continuous Flow Micro-Structured Reactors, in Micro Process Engineering, Wiley-VCH, Weinheim, 2006, pp. 173-201.

[13] R. Macey, G. Oster, Madonna 7.0, University of California, Berkeley, 1999.
[14] R. Schenk, V. Hessel, C. Hofmann, J. Kiss, H. Löwe, A. Ziogas, Numbering-up of micro devices: a first liquid-flow splitting unit, Chem. Eng. J. 101 (1-3) (2004) 421-429. 